\title{
Principles of communicating poor intrapartum events to parents and family: the SPIKE protocol
}

\section{Letter to editor}

Breaking bad news is quite frequently a tense and distressing experience for both the patient and the physician. Poor intrapartum events such as severe maternal and neonatal morbidities, neonatal malformations, perinatal death, postpartum haemorrhage, postpartum hysterectomy are certainly one of the worst situations to cope with both for the parents and family and the obstetric team.

It is important to try to define the central element of bad news (ie, to identify what makes it so bad for the patient). Basically, the impact of bad news is proportional to its effect on changing the patient's expectations: in fact, one practical definition of bad news is "any news that adversely and seriously affects an individual's view of his or her future." Messengers of bad news often inadvertently identify themselves with the negative aspects of the message. ${ }^{i}$ The reason for such coping difficulties is that pregnancy and birth are important life events, with high expectations. Nowadays the acceptance of poor intrapartum events is becoming even more difficult due to the somewhat misleading messages sent by the media which have lead common opinion to look at birth, on the one hand, as a natural, idyllic event, and on the other hand to consider poor intrapartum events as unacceptable consequences of malpractice.

A recent study revealed that $42 \%$ of physicians experience stress after breaking bad news and that the effect can last from several hours to more than 3 days. ${ }^{\text {ii }}$ Therefore, all bad news has serious adverse consequences for patients and their families. ${ }^{\text {iii,iv }}$ However poor intrapartum events do happen and they are not always necessarily the consequences of obstetric errors: they are, however, powerful stressors.

The impact of such stressors on the people involved increases as the gap between their expectations and the existing situation widens, for example, after a physiological pregnancy, the parents' expectations are usually positive and very high therefore if something goes wrong during parturition the gap between expectations and the outcome is enormous whereas during a complicated pregnancy expectations are usually less hopeful and therefore the gap may be smaller. For this, counselling may play a very important role in preparing the parents to a not necessarily positive outcome.

In this critical difficult situation: the parents and the family may be scared, confused and angry and they may not understand, or they don't want to understand what is happening and besides this they need to be understood and heard besides being "informed". The physicians may have these fears: fear of the emotional content of the news and of the possible deterioration of the patient-doctor relationship; fear of unanswerable or embarrassing questions such as "why did this happen", "why didn't anyone try to do something"; moreover in some cases we may feel the burden of the responsibility and/or of the error.

The obgyn should not only provide a technical, truthful and honest answer to the simple question: "what happened?", but should also look at the existential dimension of the subjects involved by establishing a
Volume 4 Issue 4 - 2020

\author{
E Picardo,' M Mitidieri,' C Benedetto,' S \\ Danese \\ 'Ginecologia e Ostetricia 4,A.O.U. Citta' della Salute e della \\ Scienza, Sant'Anna Hospital, Torino, Italy \\ ${ }^{2}$ Ginecologia e Ostetricia IU,A.O.U. Citta` della Salute e della \\ Scienza, Sant'Anna Hospital, Torino, Italy
}

Correspondence: E Picardo, Ginecologia e Ostetricia 4,A.O.U. Citta' della Salute e della Scienza, Sant'Anna Hospital,Torino, Italy, Email elisa.picado@gmail.com

Received: July 27, 2020 | Published: August I I, 2020

relationship based on listening, acceptance and empathy (Followfield e Jenkins, 2004).

Parents' emotional responses, which will be hard to withstand unless we have a strategy with which to address them. Without a plan , the obgyn would just be trying the best to communicate the bad news and this might not be enough. That's why there is a specific need for many physicians to receive proper and correct training and guidelines they can follow.

Physicians typically learn to communicate bad news to patients through professional experience and observation of senior physicians. Learn the son's malformation, complex genetic disease, some disability, is a painful experience well know and studied, in example: Down Syndrome, cleff lip and palate, skeletal dysplasias, spine bifide, generally congenital malformation. ${ }^{v}$ The formal researches on the difficulties that doctors find breaking bad news in general, in various field of medicine, are very few and limited to adult medicine, particulary oncology.

Results from a study published in February 2002 indicate that the communication problems of senior oncologists are not resolved with clinical experience. ${ }^{\text {vi }}$ The "SPIKES" protocolviiviii is a strategy that has been widely disseminated over the past 10 years at oncology workshops. It is not a script and is applicable in obgyn (Table 1). It highlights the most important features of a bad news interview and suggests methods of assessing the situation as it evolves and responding constructively to what happens.

This strategy provides six steps that are easy to remember: $\mathrm{S}$ stands for SETTING up the interview; P stands for PERCEPTION of the situation; I stands for INVITATION to share information; $\mathrm{K}$ stands for KNOWLEDGE of the information conveyed; E stands for EMPATHY; S stands for SUMMARY and STRATEGY for follow-up. The first step, SETTING UP of the interview the physician and his/her team should decide upon the essential information they need to convey to parents/family as well as establishing the timing of the interview 
and the setting. It is worthwhile to find a private place in order to minimize distractions. It is advisable to sit down because it gives the parents a feeling of some form of partnership in the discussion and makes level eye contact easier to achieve. Involving important third parties if desired, may help to support the parents.

Table I Strategy for breaking bad news (modified from Buckman) Meeting the above goals can be accomplished by completing six tasks, each of which is associated with specific skills

\section{Six steps of SPIKES:}

\section{S - Setting}

-Arrange for some privacy

- Involve significant others

Sit down

Make connection and establish rapport with the patient

- Manage time constraints and interruptions.

\section{P - Perception of condition/seriousness}

- Determine what the patient knows about the medical condition or what he suspects.

Listen to the patient's level of comprehension

Accept denial but do not confront at this stage.

\section{I - Invitation from the patient to give information}

Ask patient if s/he wishes to know the details of the medical condition and/or treatment

Accept patient's right not to know

Offer to answer questions later if s/he wishes.

\section{K - Knowledge: giving medical facts}

Use language intelligible to patient

Consider educational level, socio-cultural background, current emotional state

Give information in small chunks

Check whether the patient understood what you said

Respond to the patient's reactions as they occur

Give any positive aspects first

Give facts accurately about treatment options, prognosis, costs etc.

\section{E - Explore emotions and sympathize}

Prepare to give an empathetic response:

I. Identify emotion expressed by the patient (sadness, silence, shock etc.)

2. Identify cause/source of emotion

3. Give the patient time express his or her feelings, then respond in a way that demonstrates you have recognized connection between I and 2.

\section{S - Strategy and summary}

Close the interview

Ask whether they want to clarify something else

Offer agenda for the next meeting

Moreover it is important to try to reduce or eliminate the kind of body language that we often use when we are nervous and endeavour to look attentive and calm. A listening mode is very important: silence and repetition are two communication skills that will send a message to the patient that we are listening. The silence displays respect to what she or they are saying and repetitions involve using the most important words for the patient first sentence. Other basic techniques include nodding, smiling, and last but not least availability 
is essential. Adequate time for the discussion and make arrangement so that phones are answered by other staff members and to avoid interruptions are necessary. If possible, the person breaking the bad news should be a professional who has known the parents since the beginning.

The second step, PERCEPTION of the situation helps to determine what the parents know so that we can reconsider the information we had planned on giving. This step is the centre of the "before you tell, ask principles". Before breaking the bad news, we should have an accurate picture of the parent's and the family's perception of the medical situation and in particular how they view the seriousness of the condition. As the parents respond to our questions, we should take note of the language and vocabulary that they are using and be sure to use the same vocabulary in our responses. This alignment is important because it help us assess the gap between the parents expectations and the actual medical condition.

The third step, INVITATION, helps to assess how much truth the parents are able to withstand at that moment and some examples of ways to address this are: "Are you the kind of person who prefers to know all the details about this condition?"; "How much information would you like me to give you about what is going on?" LISTEN TO PARENTS CUES!

The fourth step deals with the KNOWLEDGE and the information conveyed and before we break the bad news we should give the parents a warning that bad news is coming; this gives the parents a few seconds to prepare psychologically for the bad news, examples of warning statements are: "I'm very sorry that I do not have good news for you..."; "Unfortunately I have got some bad news to tell you..." When giving the bad news we should align ourselves with the parent's level of understanding and their vocabulary. The information given should be simple, adequate, bearable, sincere, clear (avoid technical terms, abbreviations, foreign languages, euphemisms,...), essential, professional and focused on the present. The information should be given in small chunks and we should frequently check for understanding and allow for pauses and questions. Tune into the parents' readiness to hear more and know when to stop by saying, for example, "Tell me if you want or need to understand something in depth"; using indirect language as "It looks like..." instead of "Your child has..." may help.

When possible and reasonable, we should aim at keeping a right balance between truth and hope, keeping in mind that hope is not about everything turning out OK but it is about being OK with how things are. The fifth step, EMPATHY, suggests acknowledging the parents' emotions as they arise and addressing them. Emotional reaction may include feelings of unfairness and reproach, resignation, fatalism, giving up hope, anger and aggression, feelings of guilt and shame, hope, suppression of emotions... In any case, we should keep in mind that they may not react the way we expect. We should be prepared to handle both the parents' and our own emotions with EMPATHY, regarding the parents, we should observe and allow for emotional reaction, keep a kleenex handy, use touch (such as resting a hand on the parents' arm or hand if they are comfortable with this gesture) and as far as our own emotions are concerned, we should try to face and manage establishing a strong contact, without letting emotions prevail. To show our perception of the parents' emotion, it is useful to name the feelings by saying, for example: "I know, this is upsetting" to show our understanding we could say "It would be for anyone", to show our respect we may say "You're asking all the right questions", to show our support: "I'll do everything I can to help you through this". Guide and support the parents during an event that they cannot avoid facing and that they are not expected to face alone.

The last step, SUMMARY and STRATEGY for follow up means to briefly summarize the discussion, to assess level of understanding and the impact of the diagnosis on the parents. Give the opportunity to voice their concerns and questions by saying for example, what are your main concerns about this situation? Provide answers and further cognitive or emotional support if needed and offer the couple time to reflect and fully understand the meaning and implications of the information received. In case a follow up is required, it is useful to plan together the next step and last but not least end on a note of partnership and if possible, of hopefulness.

Buckman scrisse "The task of breaking bad news is a testing ground for the entire range of our professional skills and abilities. If we do it badly, the parents or family members may never forgive us; If we do it well, they will never forget us".

\section{Acknowledgments}

None.

\section{Conflicts of interest}

The author declares there is no conflict of interest.

\section{Endnotes}

i. Buckman R. Breaking bad news: why is it still so difficult? $\mathrm{Br}$ Med J. 1984;288:1597-1599.

ii. Ptacek JT, Ptacek JJ, Ellison NM. "I'm sorry to tell you..." Physicians' reports of breaking bad news. J Behav Med. 2001;24(2):205-217.

iii. Fallowfield L, Lipkin M, Hall A. Teaching senior oncologists communication skills: results from phase I of a comprehensive longitudinal program in the United Kingdom. $J$ Clin Oncol. 1998;16:1961-1968.

iv. Ptacek JT, Eberhardt TL. Breaking bad news. A review of the literature. JAMA. 1996;276:496-502.

v. Helping families deal with bad news JP Shonkoff, YE Yatchmink Behavioral and Developmental Pediatrics: A Handbook ..., 1995.

vi. Fallowfield L, Jenkins V, Farewell V, et al. Efficacy of a Cancer Research UK communication skills training model for oncologists: a randomised controlled trial. Lancet. 2002;359(9307):650-656.

vii. Baile WF, Buckman R, Lenzi R, Glober G, Beale EA, Kudelka AP. SPIKES - A six-step protocol for delivering bad news: application to the patient with cancer. Oncologist. 2000;5:302-311.

viii. Buckman R. Breaking Bad News: A Guide for Health Care Professionals. Baltimore, MD: Johns Hopkins University Press; 1992:15. 